\title{
Differential plasma microvesicle and brain profiles of microRNA in experimental cerebral malaria
}

Amy Cohen ${ }^{1}$, Anna Zinger ${ }^{1}$, Natalia Tiberti ${ }^{1,2}$, Georges E. R. Grau ${ }^{1,3+}$ and Valery Combes C $^{2,3^{*}}$

\begin{abstract}
Background: Cerebral malaria (CM) is a fatal complication of Plasmodium infection, mostly affecting children under the age of five in the sub-Saharan African region. CM pathogenesis remains incompletely understood, although sequestered infected red blood cells, inflammatory cells aggregating in the cerebral blood vessels, and the microvesicles (MV) that they release in the circulation, have been implicated. Plasma MV numbers increase in CM patients and in the murine model, where blocking their release, genetically or pharmacologically, protects against brain pathology, suggesting a role of MV in CM neuropathogenesis. In this work, the microRNA (miRNA) cargo of MV is defined for the first time during experimental CM with the overarching hypothesis that this characterization could help understand CM pathogenesis.

Results: The change in abundance of miRNA was studied following infection of CBA mice with Plasmodium berghei ANKA strain (causing experimental CM), and Plasmodium yoelii, which causes severe malaria without cerebral complications, termed non-CM (NCM). miRNA expression was analyzed using microarrays to compare MV from healthy (NI) and CM mice, yielding several miRNA of interest. The differential expression profiles of these selected miRNA (miR146a, miR-150, miR-193b, miR-205, miR-215, miR-467a, and miR-486) were analyzed in mouse MV, MV-free plasma, and brain tissue by quantitative reverse transcription PCR (RT-qPCR). Two miRNA-miR-146a and miR-193b-were confirmed as differentially abundant in MV from CM mice, compared with NCM and NI mice. These miRNA have been shown to play various roles in inflammation, and their dysregulation during CM may be critical for triggering the neurological syndrome via regulation of their potential downstream targets.
\end{abstract}

Conclusions: These data suggest that, in the mouse model at least, miRNA may have a regulatory role in the pathogenesis of severe malaria.

Keywords: Malaria, Cerebral malaria, microRNA, Disease severity, Biomarker, Microarray, Pathogenesis

\section{Background}

Malaria is a devastating infectious disease transmitted to vertebrate hosts by the bite of Plasmodium-infected female Anopheles mosquitoes [1]. Malaria remains a substantial problem affecting humans today, with approximately half of the world's population at risk. Every year, around 200 million people are infected by Plasmodium

\footnotetext{
*Correspondence: valery.combes@uts.edu.au

${ }^{\dagger}$ Georges E. R. Grau and Valery Combes are shared authors

${ }^{2}$ School of Life Sciences, Faculty of Sciences, University of Technology,

Sydney, Australia

Full list of author information is available at the end of the article
}

falciparum alone worldwide [2]. Severe malaria is most commonly caused by infection with P. falciparum, and cerebral malaria $(\mathrm{CM})$ is the most severe manifestation of malaria infection. $\mathrm{CM}$ is a syndrome characterized by unarousable coma and unconsciousness, often leading to death, or the occurrence of neurological sequelae in survivors [3]. Approximately $1 \%$ of individuals with malaria infection develop CM; however, the reason for this is currently unknown. The pathology of this syndrome is still incompletely understood, as the existing hypotheses cannot fully explain all manifestations and clinical signs of CM [4]. Other manifestations of severe malaria can 
result in severe anaemia and respiratory distress, termed here non-cerebral severe malaria (NCM) [2]. Current treatment options for $\mathrm{CM}$-that is, anti-malarial medications combined with immediate intensive care-have an $80-85 \%$ success rate. However, this is not the case in many parts of Africa, where there is increasing levels of drug resistance and, in some cases, insufficient access to adequate hospital care [5]. In fact, despite successful prevention, persisting neurological sequelae and long-term impairments are present in $25 \%$ of paediatric CM cases [6]. Therefore, further research into the pathology, progression, and treatment is essential in the prevention and elimination of malaria.

Of recent interest is the role that microvesicles (MV) play in the pathogenesis of CM both as markers of severity and effectors. Plasma MV are submicron vesicles (diameters of 200-1000 nm) released from most cell types in the microvasculature, including those involved in the pathogenesis of CM, such as platelets, erythrocytes, endothelial cells (EC), and leucocytes [7]. They form through the budding of the plasma membrane and take with them cytoplasmic proteins, lipids, and nucleic acids, as well as surface antigens from the cell of origin. Consequently, they are present in the circulation and can bind to target cells, propagating biological signals and participating in cell-cell communication [8]. While circulating MV play a role in maintaining homoeostasis at normal physiological levels [9], imbalances in their numbers have been shown to be associated with pathophysiological conditions [7]. During CM, higher levels of MV correlate with the presence of neurological symptoms, increased disease severity, and coma depth in human patients $[10,11]$ and the murine model $[7,12,13]$, while in NCM infections, the same significant increase is not observed [12,13]. In addition, blocking the production of MV, genetically [12] or pharmacologically [14], is associated with protection against the development of experimental CM. In mice, MV accumulate within the brain microvessels of infected but not healthy recipient mice, following adoptive transfer [13]. Analysis of MV, protein, lipid, and nucleic acid content provides a strong basis for studies to understand their biology and pathophysiology $[15,16]$. In disease states, MV derived from the injured organ likely contain valuable biomarkers, including miRNA, for determining the site, type, and extent of disease pathology [17].

miRNA are small, single-stranded non-coding RNA that control more than $30 \%$ of protein-coding genes, through post-transcriptional regulation of targeted gene expression and RNA silencing [18]. miRNA are known to play key regulatory roles in numerous biological processes, including cell proliferation, development, differentiation, and apoptosis [19]. miRNA were first characterized within circulating plasma vesicles by Hunter et al. [20]. Further to this, miRNA contained within MV were shown to be transferred to target cells through the circulation, suggesting that the biological effect of cells may, at least partially, depend on MV-shuttled miRNA [21].

miRNA have been shown to be dysregulated in a range of parasitic diseases [22]. Specifically, in malaria infections within mammalian hosts, several studies have explored miRNA changes before and after Plasmodium infection in different tissues, including liver and brain [23-26], and within circulating extracellular vesicles-both MV and exosomes [27-29]. Studies using the murine model have investigated the effect of Plasmodium infection on miRNA signatures within these tissues and plasma, and found that miR-16 and miR-451 decrease in abundance, while miR-27a and miR-150 increase in response to infection, and that blocking some of these miRNA confers protection to those mice. In contrast, miR-451 is increased in extracellular vesicles from infected red blood cells [27]. These changes in miRNA abundance are reversed following anti-malarial treatment [30-32]. These studies suggest that miRNA could be involved in the protective immune response or resistance against malarial infection, as well as in CM pathogenesis, depending on their downstream targets and abundance during infection. In addition, there is evidence of the impact that interactions between parasite, host, and vector can have on miRNA abundance during malarial transmission [33-36].

Despite these studies, an unbiased high-throughput characterization of miRNA abundance in MV during CM has not yet been carried out. This study examines the abundance of miRNA carried within plasma MV during experimental CM and NCM, as well as in non-infected (NI) mice. MV miRNA are then compared to those circulating in MV-free plasma and expressed in brain tissue, showing that, following infection, specific changes are observed in the three types of samples. These changes provide potential novel avenues of research into further understanding the pathogenesis of severe malaria with a particular focus on $\mathrm{CM}$, in order to harness this information for therapeutic purposes.

\section{Methods \\ Mice}

7-10-week-old CBA mice were obtained from the Animal Resource Centre (Perth) and housed under pathogen-free conditions, as per approved protocols of the University of Sydney Animal Ethics Committee (protocol number 2015/832 and 2013/5317). For experimental comparisons, three biological groups of mice were used: non-infected (NI), CM caused by infection with 
the Plasmodium berghei ANKA strain, and NCM caused by Plasmodium yoelii infection. As previously described, CBA mice are susceptible to $P$. berghei infection and succumb to $\mathrm{CM}$ during the neurological phase, between day 6 and day 14 post-infection (p.i.) [37]. Mice infected with $P$. yoelii survived beyond this point and display severe anaemia in the 3rd week after infection.

\section{Plasmodium inoculation}

All infections were initiated by intraperitoneal injection of $1 \times 10^{6}$ P. berghei or P. yoelii-parasitized red blood cells (pRBCs), as previously described [38]. Parasitaemia was monitored by counting pRBCs in Diff-Quick-stained (ProSciTech) thin blood smears by light microscopy on day 4 p.i. and every 1-2 days after this, for the duration of the infection. Mice were assessed using a clinical evaluation score, and CM diagnosed if mice presented with ruffled fur, severe motor impairment or convulsions, and were given a score of 3 or 4 as previously described [39]. $P$. berghei-infected mice were sacrificed at the time of CM (typically day 7-8 p.i.), and $P$. yoelii-infected mice without any of the signs mentioned above were classified as NCM, and sacrificed at the same time, with comparable levels of parasitaemia of approximately $7 \%$ [24].

\section{Blood sampling, MV purification, and brain tissue preparation}

Mice from all biological groups were sacrificed, and blood and brain tissue were collected. Mouse venous blood was collected by retro-orbital puncture under anaesthesia in $0.129 \mathrm{~mol} / \mathrm{L}$ sodium citrate (ratio of blood to anticoagulant 4:1). Platelet-free plasma (PFP) was prepared as previously described [13]. PFP was then centrifuged at $18000 \mathrm{~g}$ for $45 \mathrm{~min}$ and the supernatant removed and retained (MV-free plasma), while the pellet was centrifuged for a further $45 \mathrm{~min}$ at $18000 \mathrm{~g}$ in a solution of PBS and sodium citrate (ratio of PBS to anticoagulant 3:1) to pellet MV. Whole brain tissue from each mouse was placed immediately in RNAzol (Molecular Research Centre, Inc.) and homogenized. MV, MV-free plasma, and homogenized brain tissue samples were stored at $-80{ }^{\circ} \mathrm{C}$ until RNA extraction was performed. $100 \mathrm{mg}$ of homogenized brain tissue was used for RNA extractions, as per the manufacturer's instructions. For microarray analysis, MV samples were pooled during vesicle purification; all samples for RT-qPCR analysis were taken from individual mice and kept separate.

\section{RNA extraction}

Total RNA was extracted from pelleted MV, $0.4 \mathrm{~mL}$ MV-free plasma, and $100 \mathrm{mg}$ brain tissue using the commercially available protocol from Molecular Research Centre Inc. A phase separation step using 4-bromoanisole (MRC) was added to optimize RNA preparation from low amounts of starting material. All centrifugation steps were performed at $4{ }^{\circ} \mathrm{C}$, and precipitation was performed overnight at $-20^{\circ} \mathrm{C}$, with glycogen added to preserve small RNA. The concentration of RNA was determined using Nanodrop ND-1000 spectrophotometry (Nanodrop Tech), and the purity was assessed by calculation of the ratio of absorbance at 260 and $280 \mathrm{~nm}$, with a cut-off of 1.6 used. RNA concentrations of approximately $30-150,50-300$, and $3000-9000 \mathrm{ng} / \mu \mathrm{L}$ were achieved from MV, MV-free plasma, and brain tissue, respectively.

\section{cDNA synthesis and preamplification}

For OpenArray analysis the starting RNA concentration was set at $100 \mathrm{ng}$; for RT-qPCR analysis, it was $10 \mathrm{ng}$. For both, preamplification was used to increase the amount of cDNA. cDNA synthesis and preamplification were performed using commercial rodent kits (Megaplex RT and preamplification kits) and rodent Megaplex ${ }^{\text {TM }}$ Primer Pools for OpenArray, or custom primer pools of the targets of interest described below, for RT-qPCR (ThermoFisher Scientific).

\section{OpenArray analysis}

Pre-amplified samples were loaded onto TaqMan OpenArray MicroRNA Panels to be run on the QuantStudio 12K Flex system, as per the manufacturer's instructions (ThermoFisher Scientific). 754 rodent miRNA, consisting of validated mature mouse and rat miRNA (Sanger miRBase release v.15), were amplified in each sample, with technical replicates, together with internal controls. QuantStudio and ExpressionSuite Analysis software (ThermoFisher Scientific), utilizing the comparative $(\Delta \Delta)$ $\mathrm{C}_{\mathrm{T}}$ method, were used to quantify relative expression across all miRNA and samples with relative quantification values expressed as CM/NI. Global normalization was performed using the global mean CT value of targets common to every sample as the normalization factor, on a per sample basis-the recommended and most robust strategy for normalization in large scale $(>384)$ miRNA expression profiling studies [40]. To reduce false positive results, $C_{R T}$ (cycle of relative threshold) values were filtered to remove values over 30 , considered not to be true results. In addition, technical replicates with a standard deviation (SD) over 0.5 between $C_{R T}$ values, and with an amplification score below 1.2 (low quality) were removed. Lastly, miRNA amplified in less than $50 \%$ of biological replicates were considered as lowly expressed and excluded from the analysis. Relative quantification was calculated using algorithms included in the ExpressionSuite analysis software. Statistical calculations were 
performed using multiple $t$ tests-one per row, with fewer assumptions.

\section{Pathway analysis}

Microarray results were analyzed with DIANA miRPath version 3 [41] and Ingenuity ${ }^{\circledR}$ Pathway analysis-IPA (Ingenuity ${ }^{\circledR}$ System, http://www.ingenuity.com) to combine filtering and miRNA matching to mRNA targets in order to provide insight into the potential biological effects of miRNA and to predict their role within target pathways. This software uses TarBase version 7 [42], which houses a manually curated collection of experimentally tested miRNA targets. The input dataset was compared to all available biological pathways provided by the Kyoto Encyclopaedia of Genes and Genomes (KEGG) [43], with significantly influenced pathways identified $(\mathrm{P}<0.05)$ and a False Discovery Rate of $5 \%$. The database was searched with the full names of each murine miRNA as per the ThermoFisher Scientific product information and miRBase version 21: mmu-miR-16-1-3p, mmu-miR21a-3p, mmu-miR-146a-5p, mmu-miR-150-5p, mmumiR-193b-3p, mmu-miR-205-5p, mmu-miR-215-5p, mmu-miR-297a-3p, mmu-miR-328-3p, mmu-miR335-3p, mmu-miR-467a-5p, mmu-miR-486a-5p, mmumiR-685, mmu-miR-1949, and rno-miR-10b-5p.

\section{RT-qPCR analysis}

RT-qPCR results often depend on the quality of the endogenous control genes chosen, ideally demonstrating gene expression that is constant and highly abundant across tissues and cell types, and across changing biological conditions or treatments. To verify these miRNA of interest highlighted by the OpenArray, four control miRNA (U6, Y1, sno-RNA-135, and sno-RNA-202-the murine miRNA from the original control panel from the OpenArray) were tested for their robustness of normalization, and their expression across all biological groups and sample types. RT-qPCR analysis was performed using the Taqman gene expression master mix, following recommended protocols. The starting RNA concentration was set at $10 \mathrm{ng}$ and each sample was tested in duplicate. Taqman small RNA assays for the miRNA of interest, i.e. hsa-miR-146a, hsa-miR-150, hsa-miR-205, hsa-miR-486, mmu-miR-10b, mmu-miR-193b, mmumiR-215, mmu-miR-467a, two miRNA amongst those unique to either CM or NI MV i.e. hsa-miR-590-5p and rno-miR-450, and the four controls were used. Replicates for which the SD was $>0.5$ were removed. Statistical calculations were performed using a non-parametric Kruskal-Wallis test, and a two-stage post hoc test to correct for multiple comparisons by controlling the False Discovery Rate for significant results. Results were considered significant, if $\mathrm{P}<0.05$.

\section{Sample size calculation}

A sample size calculation was performed using GraphPad Statmate software v2.0. As this formula required a known SD, the OpenArray results were used to inform the calculation of a sample size necessary for subsequent RT-qPCR verification. A significance level (alpha) of 0.05 (two-tailed) and a 90\% power were chosen as the parameters for this calculation in order to increase the stringency, and decrease the chance of false-positive findings for this verification analysis. It was found that a sample size of 5 in each group was sufficient to detect a significant difference between means of $\geq 5.93$.

\section{Results}

\section{Experimental design}

To examine the abundance of the miRNA cargo of MV released during $\mathrm{CM}$, compared with NI conditions, the OpenArray system (ThermoFisher Scientific) was used. The targets of interest found in the initial screening process were verified using qPCR analysis: examining MV, MV-free plasma, and brain tissue from NI, NCM and $\mathrm{CM}$ mice. miRNA of interest were evaluated in relation to $\mathrm{CM}$ pathogenesis using target prediction and pathway analyses. A graphical representation of the experimental design used is shown in Fig. 1.

\section{Distinct profile of miRNA within MV in CM compared with NI mice, using array analysis}

The abundance of miRNA contained in MV purified from PFP obtained from NI and CM CBA mice were compared using OpenArray ${ }^{\circledR}$ analysis. For microarray analyses, three groups of NI mice and four groups of CM mice were used, with five samples pooled per group. A distinct abundance profile was observed in the MV from CM compared with NI mice. Within each biological group, approximately $60 \%$ of miRNA were detected in all replicates within a biological group, and 408 and 361 miRNA were detected in $>50 \%$ of NI (blue, vertical stripes) and $\mathrm{CM}$ replicates (red, vertical stripes), respectively, and were therefore studied further. 431 miRNA were quantified in at least one biological group (dark grey) and 348 miRNA were common to both biological groups (differential, light grey), while 60 and 23 miRNA were detected only in NI (blue) or CM MV (red), respectively (Fig. 2a).

\section{Differentially abundant miRNA play potential roles in malaria infection and inflammation}

miRNA that were present in $>50 \%$ of replicates within both $\mathrm{NI}$ and $\mathrm{CM}$ groups in the microarray analysis were further analyzed to identify significant miRNA of interest (differential miRNA from Fig. 2a, $n=348$ ). A cut-off of \pm twofold was used to compare the relative expression of these miRNA between the groups, measured as a ratio of 


\section{Sample preparation}

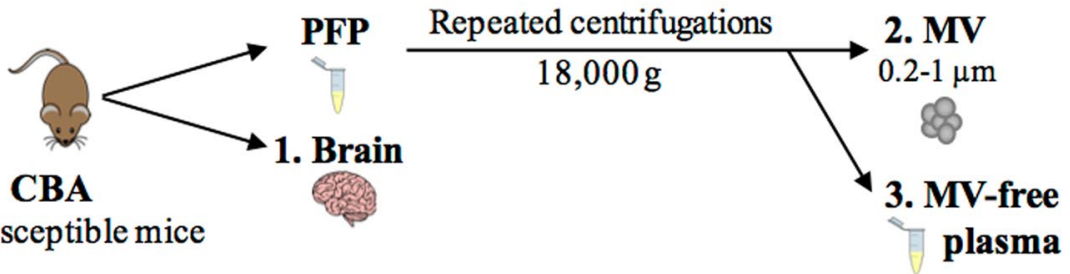

\section{miRNA cargo investigation}
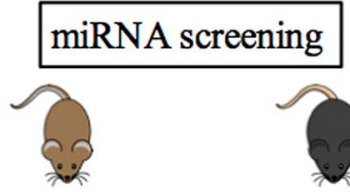

Non-infected

(NI)

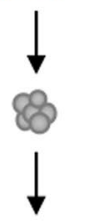

RNA extraction, reverse transcription \& preamplification

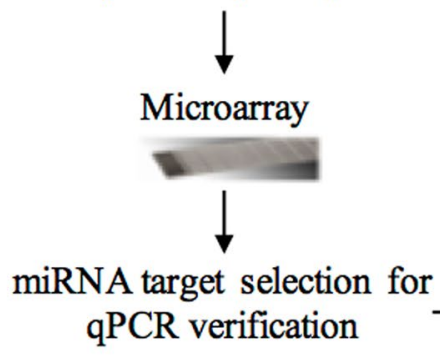

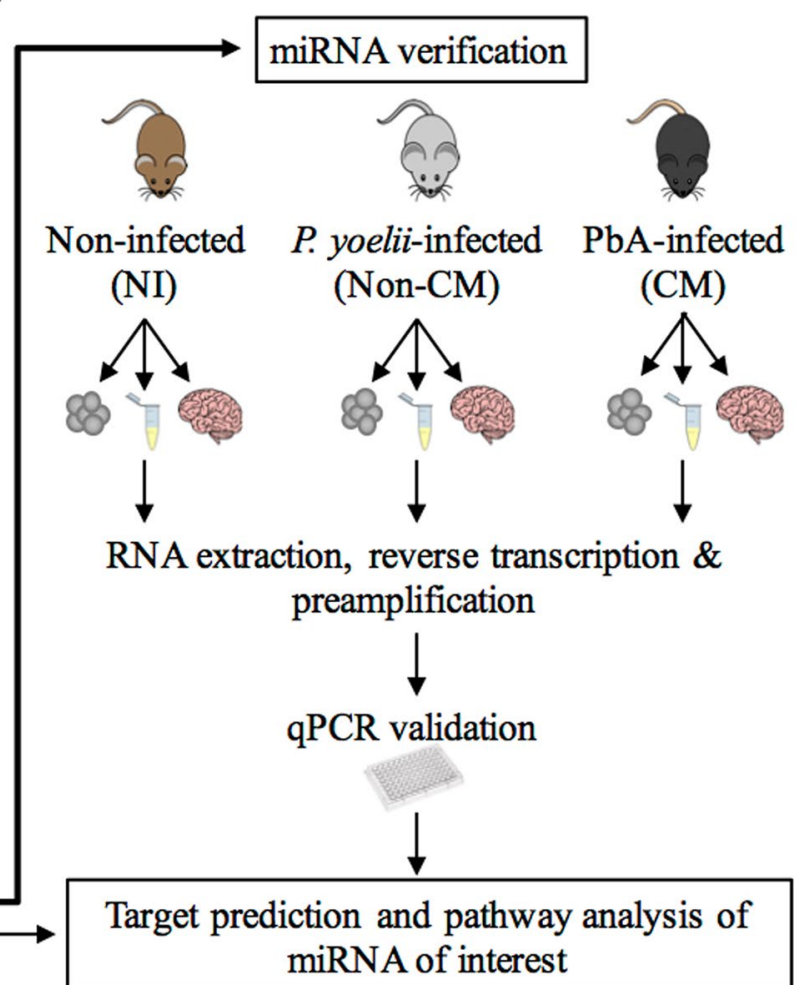

Fig. 1 Experimental design. Graphical summary of the experimental design applied in the present study. PFP platelet-free plasma, MV microvesicle, NI non-infected, CM cerebral malaria. Images were obtained from Pixabay.com and ChemDraw (PerkinElmer)

$\mathrm{CM} / \mathrm{NI}$. Figure $2 \mathrm{~b}$ displays the results in a volcano plot, where miRNA common to both biological groups were plotted according to their fold change, and the significance of that association. Four miRNA, shown in green, showed significantly decreased abundance in CM conditions, and 11 miRNA (red) were significantly increased compared with NI conditions. These miRNA of interest were further investigated and shown to have diverse roles defined in the literature, and using DIANA miRPath software. 19 pathways were significantly regulated by the chosen miRNA of interest, as detailed in Additional file 1: Table S1 and Additional file 2: Table S2, and include those relating to Prion diseases $\left(\mathrm{P}=2.2 \mathrm{e}^{-20}, 7\right.$ genes controlled by 5 miRNA in pathway), regulation of actin cytoskeleton $(\mathrm{P}=0.02$, 41 genes by 8 miRNA), gap junctions $(\mathrm{P}=0.04$, 14 genes by 5 miRNA), Chagas disease $(P=0.04,21$ genes by 6 miRNA), and toxoplasmosis $(P=0.04,22$ genes by 8 miRNA). Interestingly, these miRNA strongly regulate other neurological conditions, pathways related to MV formation, or other parasitic diseases, further highlighting their potential importance in CM. Of the 15 miRNA identified by OpenArray analysis as significantly differentially abundant, eight of them were chosen for verification by RT-qPCR, according to their postulated roles in $\mathrm{CM}$ and other similar neurological or inflammatory 

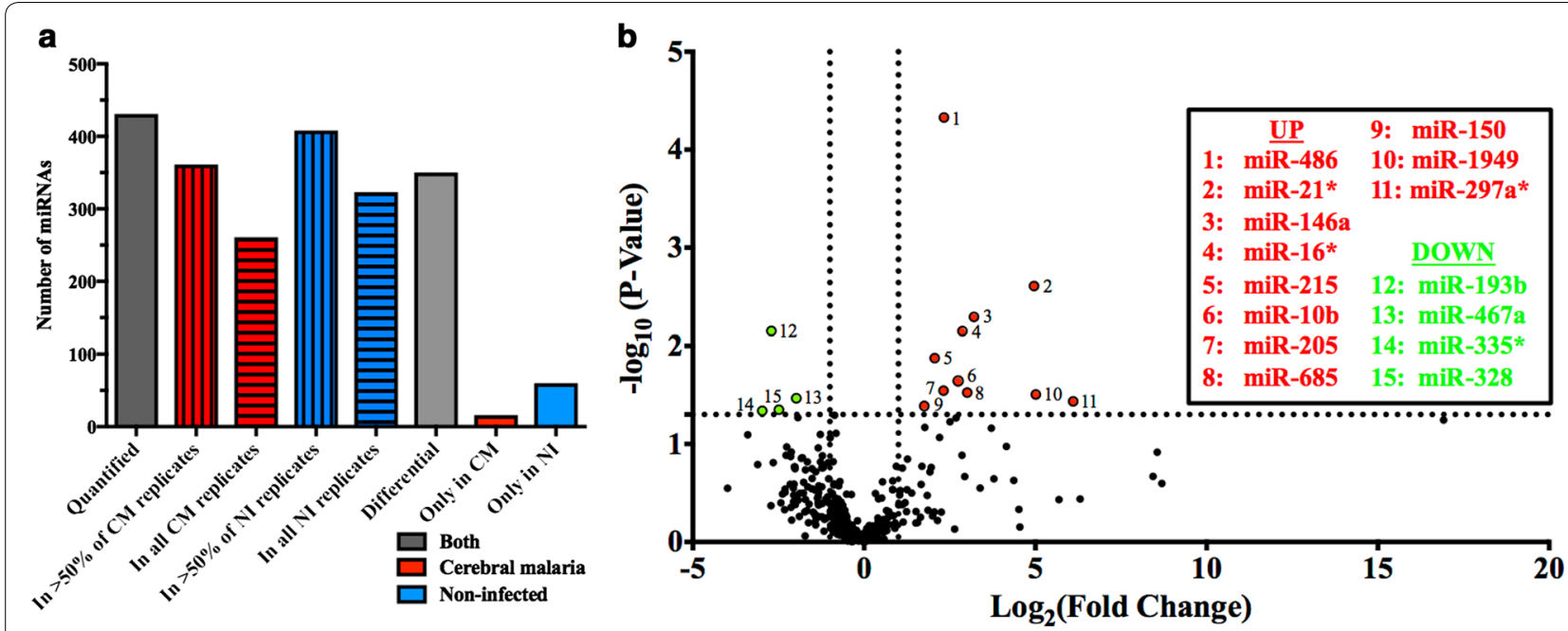

Fig. 2 Detectable miRNA profiles in MV from NI compared with CM CBA mice. a The number of miRNA quantified in one or more biological group, and in $>50$ or $100 \%$ of replicates in each biological group are shown. miRNA present in $>50 \%$ of replicates are further separated into groups of those common to both biological groups (further examined in Fig. 1b) and those expressed in one biological group only. b The volcano plot displays miRNA present in both biological groups; for each miRNA, the fold change $\left(\log _{2} \mathrm{FC}\right)$ and the significance $\left(-\log _{10} \mathrm{P}\right.$-value) are reported. P values were obtained with a student's $t$ test, with fewer assumptions. Horizontal threshold line: $P=0.05$, a higher value indicates greater significance; vertical threshold lines: fold change $=-2$ (left) and 2 (right). Highlighted in red are miRNA that are significantly increased in abundance in CM conditions, and in green, decreased

conditions, as well as their regulation of genes within the malaria pathway.

\section{Choice of reference miRNA candidates for RT-qPCR normalization}

Before validating these miRNA of interest by RT-qPCR, potential control miRNA were assessed, in order to determine the best normalization strategy to be applied to RT-qPCR results. Four candidate controls-U6, Y1, sno-RNA-135, and sno-RNA-202-were investigated on the OpenArray results. In particular, the global normalization strategy was compared to U6, and to two different combinations-one including U6 and one not (Fig. 3), as the validity of $\mathrm{U} 6$ as a control has previously been debated [44]. Candidate control miRNA were deemed suitable if they were detected in over $70 \%$ of samples, and if the $C_{R T}$ values fell within the range of $18-28$, as per previous rigorous testing $[45,46]$, and all four fulfilled these requirements (Fig. 3a). The number and type of miRNA found to be significant with the four different normalization strategies were then compared (Fig. 3bd), and increasing numbers of significant miRNA were observed using fewer candidate control miRNA (Fig. 3b). Of the 15 miRNA found to be significantly differentially abundant using global normalization, the numbers of miRNA remaining significant, decreased with the numbers of control miRNA used in the panel, i.e., 12 miRNA with 4 controls, 11 with 3 controls and 7 with U6 alone
(Fig. 3c, d). Importantly, of the eight miRNA from this list of 15 that were chosen for further validation by RTqPCR, 7, 6 and 4 were also found to be significant when normalizing with the respectively listed groups above (Fig. 3c). Therefore, the panel of four control miRNA was chosen to normalize RT-qPCR verification results, as this strategy was found to be the most similar to global normalization.

\section{Significantly changed abundance of miR-146a and miR-193b in MV following $P$. berghei infection}

Verification by RT-qPCR was performed on newly generated samples. Seven of the 15 miRNA selected from the OpenArray were removed from the verification due to a lack of validated targets and this will be further developed in the discussion (Table 1, miR-16*, miR-21*, miR297a*, miR-335*, miR -328, miR-685, and miR-1949. Two additional miRNA-miR-590-5p and miR-450-were selected among those unique to either CM or NI MV for verification; however, as amplification was not adequate across all samples and biological groups, data for these two miRNA as well as for miR-10b, were not shown (Table 1). All the remaining miRNA (Table 1, miR-146a, miR-150, miR-193b, miR-205, miR-215, mir-467a, and miR-486) were tested on MV samples as per the OpenArray analysis and also on MV-free plasma and brain tissue from NI, NCM, and CM mice. This provided a more comprehensive analysis of the changes occurring 


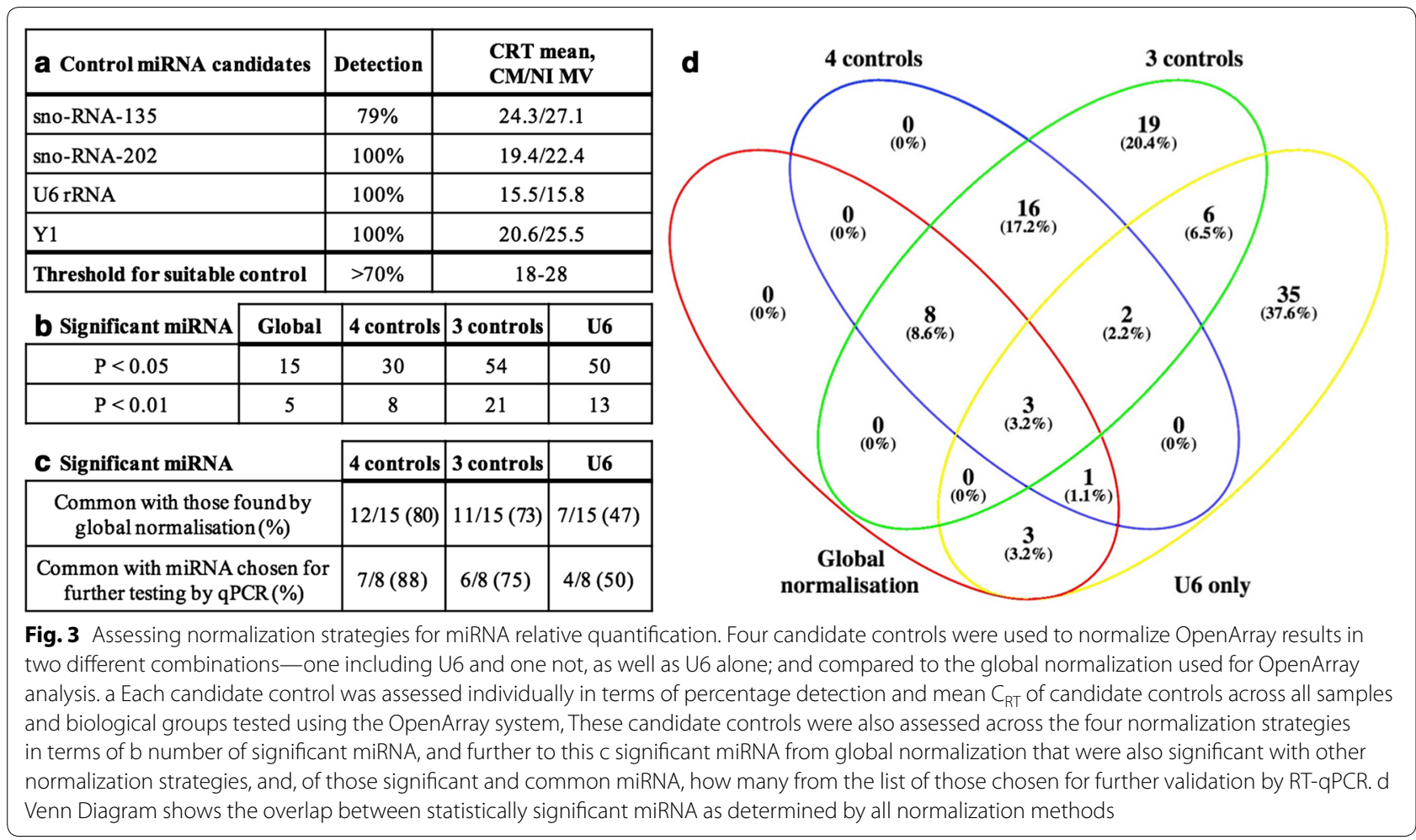

during Plasmodium infection in order to identify those specific to neurological complications, in comparison to non-cerebral infections or non-infected status. For RTqPCR analysis, five mice from each of the three biological groups were used, with each mouse representing an individual sample. All selected miRNA were expressed at detectable levels in all samples under normal physiological conditions, but their expression levels after the inoculation of P. berghei (CM) or P. yoelii (NCM) demonstrated altered abundance in $95 \%$ of cases. Significant changes were found in the abundance of miR-146a, miR-193b, miR-205, miR-215, and miR-467a, as shown in Fig. 4. miR-146a was significantly more abundant in $\mathrm{MV}$ and MV-free plasma from mice at the time of CM $(\mathrm{P}=0.027$ and 0.005 , respectively), compared with that of NI mice, and in MV-free plasma from NCM mice compared with NI mice $(\mathrm{P}=0.012)$, while no significant differences were observed in the brain samples. In MV samples only, miR-193b was less abundant at the time of $\mathrm{CM}$, compared with both NI $(\mathrm{P}=0.007)$ and NCM conditions $(\mathrm{P}=0.007)$ (Fig. 4). The significant changes in miRNA abundance in MV reflected the findings of the OpenArray. Specifically, miR-146a and miR-193b were both significantly dysregulated in the MV from CM mice compared with NI-increasing 3.2- or 7.2-fold in the case of miR-146a, or decreasing 2.7- or 7.5-fold (miR-193b) in array and RT-qPCR analysis, respectively (Table 1 ).
miR-467a was significantly less abundant in the MVfree plasma from $\mathrm{CM}$ mice $(\mathrm{P}=0.050)$ and $\mathrm{NCM}$ mice $(\mathrm{P}=0.020)$ compared with NI (Fig. 4). Interestingly, different profiles were observed in the brain samples: miR205, miR-215, and miR-467a were significantly increased abundance in the brains of CM mice compared with NI $(\mathrm{P}=0.011,0.016$, and 0.015 respectively), but no change was observed in the brains of NCM mice (Fig. 4).

\section{No significant change in the abundance of miR-150, miR-205, miR-215, miR-467a, and miR-486 in MV following Plasmodium infection}

Of the seven miRNA of interest tested by RT-qPCR, miR$146 \mathrm{a}$ and miR-193b showed the same significant change in abundance as in the OpenArray (from Fig. 2b). A further four miRNA-miR-150, miR-215, miR-467a, and miR-486 showed the same directional change in abundance as in the OpenArray analysis, without reaching significance (Fig. 4). Comparative results for the miRNA of interest for the OpenArray and RT-qPCR analyses are displayed in Table 1. miR-205 showed a small decrease in abundance in the RT-qPCR validation experiments, compared to the significantly increased abundance in the OpenArray analysis. miR-10b, results were not shown, due to poor RT-qPCR amplification across all sample types. The direction of regulation in CM conditions was the opposite for MV and brain tissue in the case of 
Table 1 Choice of targets for validation by RT-qPCR from OpenArray analysis

\begin{tabular}{|c|c|c|}
\hline \multirow[t]{2}{*}{ miRNA } & \multicolumn{2}{|c|}{$\begin{array}{l}\text { Significance of fold change in CM vs. } \\
\text { NI MV }\end{array}$} \\
\hline & OpenArray & RT-qPCR \\
\hline hsa-miR-328 & $-2.5^{*} \pm 0.93$ & Not tested ${ }^{\mathrm{a}}$ \\
\hline hsa-miR-335* & $-3.0^{*} \pm 1.13$ & Not tested ${ }^{\mathrm{a}}$ \\
\hline mmu-miR-16* & $2.8^{* *} \pm 0.65$ & Not tested ${ }^{\mathrm{a}}$ \\
\hline mmu-miR-21* & $5.0^{* *} \pm 0.88$ & Not tested ${ }^{\mathrm{a}}$ \\
\hline mmu-miR-297a* & $5.8^{*} \pm 1.60$ & Not tested ${ }^{\mathrm{a}}$ \\
\hline mmu-miR-685 & $3.0^{*} \pm 1.00$ & Not tested ${ }^{\mathrm{a}}$ \\
\hline mmu-miR-1949 & $5.0^{*} \pm 1.69$ & Not tested ${ }^{\mathrm{a}}$ \\
\hline hsa-miR-590-5p & Unique to $\mathrm{NI}$ & Not validated $^{b}$ \\
\hline rno-miR-450 & Unique to CM & Not validated ${ }^{b}$ \\
\hline mmu-miR-10b & $2.7^{*} \pm 0.85$ & Not validated ${ }^{b}$ \\
\hline hsa-miR-146a & $3.2^{* *} \pm 0.68$ & $7.2^{*} \pm 2.74$ \\
\hline hsa-miR-150 & $1.8^{*} \pm 0.64$ & 2.7 (ns) \pm 2.26 \\
\hline hsa-miR-205 & $2.3^{*} \pm 0.75$ & -0.5 (ns) \pm 1.89 \\
\hline hsa-miR-486 & $2.3^{* * *} \pm 0.18$ & 4.7 (ns) \pm 1.45 \\
\hline mmu-miR-193b & $-2.7^{* *} \pm 0.62$ & $-7.5^{*} \pm 062$ \\
\hline mmu-miR-215 & $2.1^{*} \pm 0.55$ & 4.6 (ns) $\pm 99.39^{c}$ \\
\hline mmu-miR-467a & $-2.0^{*} \pm 0.69$ & $-5.6(n s) \pm 0.96$ \\
\hline
\end{tabular}

The list of significantly differentially expressed miRNA in CM vs NI MV from the OpenArray analysis was compared with the results obtained by RT-qPCR analysis. Results presented as relative quantification \pm SEM

$P:{ }^{*}=0.05-0.01,{ }^{* *}=0.01-0.0001,{ }^{* * *} \leq 0.0001$

a miRNA not validated by RT-qPCR due to lack of relevance to this study

${ }^{b}$ miRNA not validated despite being tested by RT-qPCR due to technical issues

c Large SEM variation due to outliers in $\mathrm{NI}$ and CM MV groups

miR-150, miR-205, miR-193b, and miR-467a. For these 4 miRNA as well as miR-146a, the direction of regulation in CM conditions was the same for MV and MV-free plasma. In all cases within the brain samples-except miR-150-miRNA abundance increased (significantly or not, in the case of specific miRNA) in CM compared with $\mathrm{NI}$ and NCM conditions, with NCM being an intermediate abundance level between the other two biological groups. This intermediate abundance in NCM conditions was applicable in almost all miRNA tested in MV and MV-free plasma samples as well.

Significantly differentially abundant miRNA regulate genes with identified roles during malaria infection

Of the differentially abundant miRNA from the OpenArray analysis that were further analyzed by RT-qPCR, five significantly abundant miRNA regulate genes within the KEGG malaria pathway (Fig. 5). This figure summarizes the changes occurring during malaria infection at the gene level, and the impact this has on various cell types and molecules produced. This figure has been revised from the original KEGG pathway, with genes involved highlighted based on the miRNA of interest and their roles in regulating these genes. Within the malaria pathway, a collection of miRNA specifically regulate the groups of genes surrounding MV release, regulation of inflammatory cytokines, and cytoadherence of cells to the vascular endothelium. To summarize, three miRNA: miR-146a, miR-193b, and miR-467a, control 10 genes within the malaria pathway: $\mathrm{CD} 40$ ligand $(\mathrm{CD} 40 \mathrm{~L}$, CD154), CXCL8 (IL-8), IFN $\gamma$, Integrin $\beta 2$ (ITG $\beta 2$ ), Transforming Growth Factor $\beta 2$ (TGF $\beta 2$ ), Thrombospondin genes (THBS1/TSP1, THBS2/TSP2), Toll-Like Receptors (TLR2, TLR4), and TNF. Further to this, many of these miRNA also have roles defined in the literature, relating to similar neuro-inflammatory conditions (more detail in Additional file 1: Table S1).

\section{Discussion}

As miRNA research has expanded into numerous disease areas, it has become clear that expression levels of certain miRNA are altered in many diseases, including malaria [20, 24, 26]. However, despite these studies examining miRNA during malaria infection, the mechanism of action of miRNA and whether they play a pathogenic or immuno-protective role is still unclear. This study describes for the first time, the changes in miRNA content of MV during Plasmodium infection in mice and shows a clear dysregulation of miRNA abundance within circulating MV during experimental $\mathrm{CM}$ as compared with non-infected and non-CM mice.

In mice, $P$. berghei infection triggers an intricate series of events that leads to $\mathrm{CM}$, including an increased release of extracellular vesicles, released from EC, platelets, and RBCs [12, 13]. miRNA have also been implicated in this process by actively regulating the immune or inflammatory response. In fact, many studies have shown that miRNA constitute a key mechanism by which MV mediate cellular communication. Specifically, during malaria infections, miRNA from $\mathrm{pRBC} M V$ have been shown to modulate EC permeability, affecting vascular function [27]. Furthermore, evidence of changed miRNA levels in the brains of mice with CM compared with NCM or NI mice has been reported [24, 26]. Using OpenArray technology as a screening approach, 754 miRNA targets were assessed and 15 miRNA with significantly dysregulated abundance in MV from CM mice in comparison to NI mice were detected. To verify the screened results, and to better understand miRNA changes during the severe infection, eight of these targets were selected and their abundance measured by RT-qPCR in MV, MVfree plasma, and brain tissue from CM and NI mice. The remaining seven miRNA were not tested as they had no validated murine targets following IPA analysis and/or few or no publications were available examining them. 


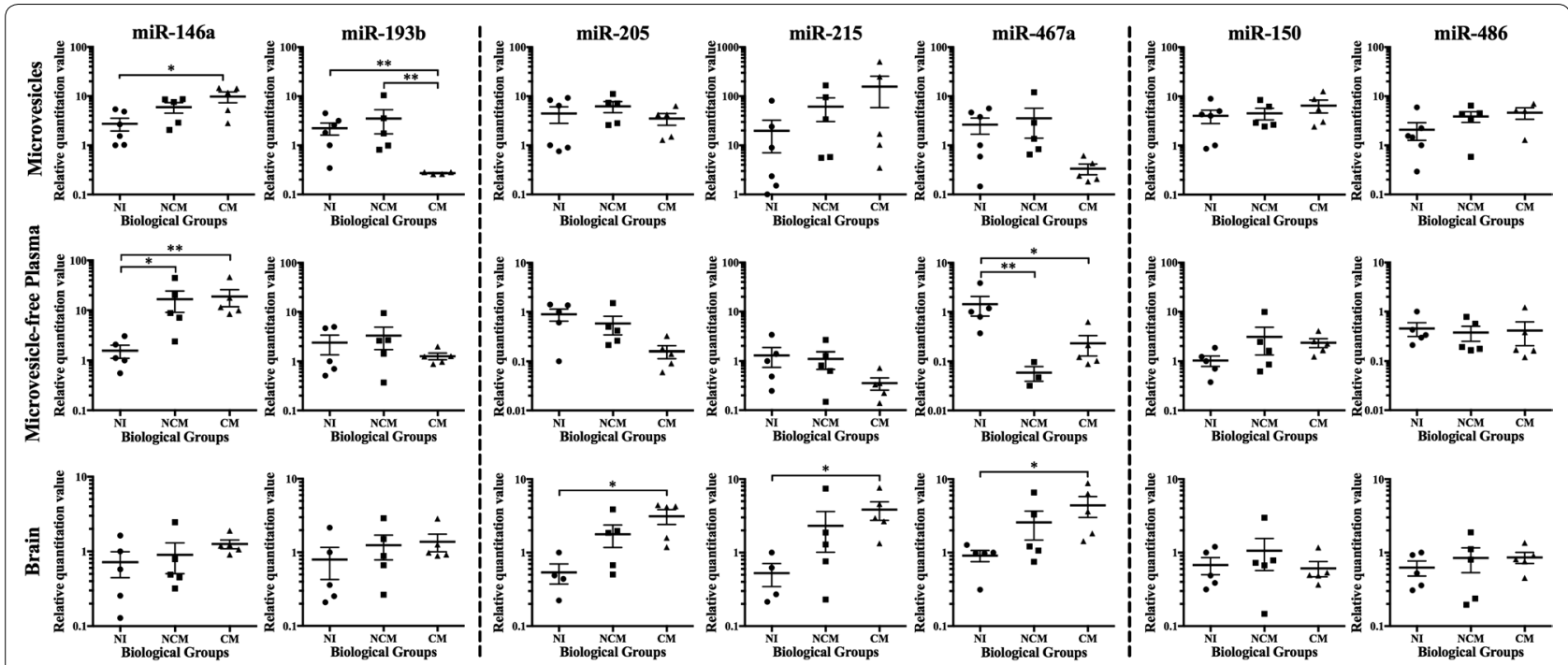

Fig. 4 Changes in abundance of miRNA of interest in MV, MV-free plasma and brain tissue. Dot plots show the expression levels of miR-146a, miR-193b, miR-205, miR-215, miR-467a, miR-150, and miR-486 measured for MV, MV-free plasma, and brains from NI, NCM, and CM conditions, expressed as normalized values as compared to the expression of a panel of control miRNA in each case. The results are presented as follows: significant changes in MV - miR-146a and miR-193b, significant changes in the brain-miR-205, miR-215, and miR-467a, no significant changesmiR-150 and miR-486. miR-146a and miR-467a also showed significant changes in the MV-free plasma. The horizontal line denotes the mean value, and the error bars denote SEM. A nonparametric Kruskal-Wallis test was carried out. If the Kruskal-Wallis test was significant, post hoc tests-Dunn's multiple comparisons test - were carried out. The results of these are denoted as ${ }^{*}=0.05-0.01,{ }^{* *}=0.01-0.0001,{ }^{* * *} \leq 0.0001$

In contrast, the remaining miRNA of interest chosen for RT-qPCR verification did have validated murine targets, as well as relevant roles related to neuropathologies and inflammatory conditions similar to CM (Additional file 2: Table S2). To assess the results regarding their specificity to the malarial neurological syndrome, samples obtained from NCM mice were also analyzed.

Upon verification, two miRNA were confirmed as significantly dysregulated in CM MV: miR-146a and miR193b, suggesting a role for these miRNA in cerebral pathology, as the same significant changes were not observed in NCM mice [47]. miR-146a was significantly over-abundant in CM MV and MV-free plasma compared to NI, but not in brain tissue-although the same trend in abundance shift was observed. An increased abundance of miR-146a is triggered by inflammatory factors including IL-1 and TNF [48], and dysregulates several targets, the majority of which are involved in toll-like receptor pathways, triggering a cytokine response, or in the innate immune system $[48,49]$, and these changes are also observed in CM [50]. Within the malaria pathway, miR-146a suppresses the CD40L, CXCL8, IFNy, TLR2, TLR4, and ITG $\beta 2$ genes. Together with miR-155 and miR-21, miR-146a operates in a negative feedback loop to specifically calibrate inflammatory responses [51]. The role this miRNA plays within inflammatory conditions [49] has been researched extensively, identifying miR146a as a biomarker for sepsis [52] and prion infections
[53]. Notably, in the latter, miR-146a was part of a miRNA signature within exosomes from prion-infected neuronal cells. Finally, miR-146a has also been implicated in the overactive response to infection, therefore has previously been proposed to have potential as a therapeutic target [54].

Remarkably, miR-193b was significantly decreased in CM MV as compared not only to NI, but also NCM MV. Critically, no similar significant difference was observed in the MV-free plasma or brain tissue, though the opposite trend in abundance shift was observed in the latter. miR-193b plays a role in the TGF $\beta 2$ signaling pathway [55], and in monocyte-macrophage differentiation [56], both of which have been explored in relation to CM [50]. Within the malaria pathway, miR-193b promotes the THBS1, THBS2, and TGF $\beta 2$ genes; and regulates apoptosis by targeting myeloid leukaemia cell differentiation protein 1, among others [57]. Notably, miR-193b is a predictor of mortality in several other severe inflammatory conditions with associated neurotropic complications. Specifically, miR-193b is decreased in abundance in amyotrophic lateral sclerosis [58], and in sepsis [59], for which it has been identified as a biomarker.

Interestingly, miR-146a and miR-467a were significantly increased and decreased, respectively, in both $\mathrm{CM}$ and NCM MV-free plasma compared to NI. Therefore, these miRNA are likely to be indicative of a severe malaria infection, rather than specific to neurological 


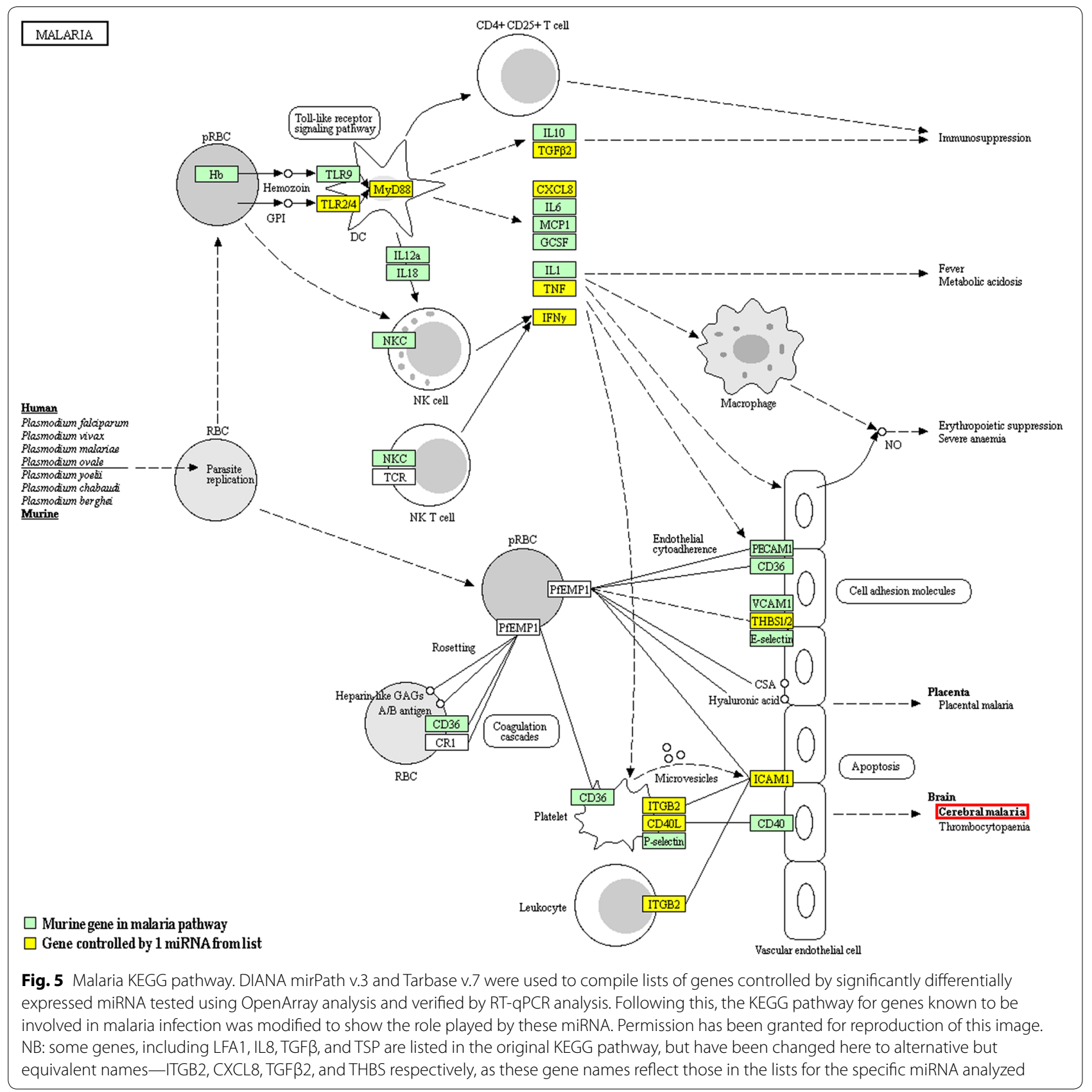

complications. In contrast to the changes observed in MV, miR-205, miR-215, and mir-467a were increased only in the brains of CM compared with NI mice, or NCM mice. These data support the observation that particular miRNA may be detectable only in specific samples, suggesting a preferential expression from specific cell types [60, 61].

The differences in abundance profiles observed in the three different sample types analyzed provide further evidence that miRNA appear to be selectively expressed in the circulation, whether in the MV-free plasma itself, or expressed on the surface of or packaged within circulating MV. Specifically, the directional change in abundance of these miRNA of interest in MV-free plasma in $\mathrm{CM}$ conditions more closely reflected the changes within MV, compared with the opposite changes within brain tissue. In examining further the brain, all but one miRNA (miR-150) showed increased abundance in the brains of CM mice, as compared with their NI 
counterparts; interestingly their NCM counterparts demonstrated intermediate levels of expression, further demonstrating the difference in the degree of in severity between the two infections.

In line with previous studies comparing plasma or serum, and MV, MV-free plasma samples showed a more variable expression pattern for the miRNA of interest [62]. With the MV portion of the MV-free plasma removed, remaining miRNA are likely to be stable circulating extracellular miRNA secreted from cells, or present in other types of vesicles not tested in the current analysis-such as exosomes, as both of these sources have previously been identified in other diseases or conditions [27, 63]. Previous studies have identified miRNA present in a range of fluids and biological specimens or tissue types. Among these profiles, some highly abundant miRNA were common to multiple sample types, while others were enriched only in particular conditions [61], and the same phenomenon was observed in this study. The difference in abundance of particular miRNA in particular sample types here indicates the variety of the roles played by certain miRNA in specific locations or conditions.

Those miRNA validated by RT-qPCR and displaying significant differences in abundance during CM compared with NI conditions were examined within the KEGG pathway for malaria (Fig. 5), which highlights evidence of miRNA regulation relating to many of the critical components in CM development. Specifically, during severe Plasmodium infection, miR-146a and/or miR193b regulate functions such as production of inflammatory cytokines (also involving miR-467a), recruitment of macrophages, increased nitric oxide production, disruption of neuronal function, as well as modulation of the expression of surface molecules controlling interactions between circulating cells and endothelial cells $[4,64]$.

Of importance to this work are the recent improvements in the understanding of the role of miRNA in the regulation of molecular and cellular networks associated with inflammation [65]. Consequentially, miRNA potential as biomarkers of inflammatory processes and infectious diseases has become increasingly evident [65]. In this context, further investigations of miRNA contained in MV (such as miR-146a and miR-193b) or circulating in the MV-free plasma (such as miR-146a and miR-467a), as well as the remaining miRNA of interest from the OpenArray not yet verified by RTqPCR, such as miR-328 and miR-335", are necessary to assess whether these miRNA are biomarkers of severity or mortality. Detailed examination of the kinetics of these miRNA in MV over the course of infection could reveal novel candidate markers for the early prognosis or diagnosis of CM. Furthermore, the evaluation of their abundance in human paediatric samples as well as functional studies using in vitro CM models to establish their cellular source and targets, will help to understand the clinical potential of these miRNA and their role in $\mathrm{CM}$ pathogenesis. It is also important to note that the OpenArray identified a significant number of miRNA expressed only in CM or NI MV, that warrant further investigation as to their biological relevance. In investigating for the first time the miRNA content of murine MV during CM, these results indicate that the content of plasma MV is affected during the severe infection and should be considered in the understanding of CM pathogenesis.

\section{Additional files}

Additional file 1: Table S1. Pathways significantly regulated by miRNA of interest. The list of significantly differentially expressed miRNA from the OpenArray analysis was analyzed using miRPath software to determine the significantly regulated pathways they controlled. The KEGG pathway identification and $\mathrm{P}$ value of each pathway is shown, as well as the number of genes within each pathway controlled by a number of miRNA from our list of miRNA of interest.

Additional file 2: Table S2. Significantly differentially expressed miRNA of interest. The list of significantly differentially expressed miRNA from the OpenArray analysis was analyzed using miRPath software to determine their proposed roles and downstream targets. The directional change in regulation of each miRNA in CM as compared to NI MV is shown. These ten miRNA were chosen for further study based on their identified roles in the literature: eight are significantly differentially expressed, and two were selected from the populations of miRNA unique to each biological group. Here, $P$ value thresholds of significance as defined previously.

\section{Authors' contributions}

$A C, V C$ and GEG designed the study. AC performed the experiments and data analyses, and wrote the manuscript. AZ provided technical expertise and assistance in the setup of microarray analysis. NT contributed part of the statistical analyses. All authors provided advice relating to study design, figure preparation, and manuscript revision. All authors read and approved the final manuscript.

\section{Author details \\ ${ }^{1}$ Vascular Immunology Unit, Department of Pathology, The University of Syd- ney, Sydney, Australia. ${ }^{2}$ School of Life Sciences, Faculty of Sciences, University of Technology, Sydney, Australia. ${ }^{3}$ La Jolla Infectious Diseases Institute, San Diego, CA, USA.}

\section{Acknowledgements}

The authors would like to acknowledge the Bosch Institute Molecular Biology Facility, where microarray experiments were conducted. We furthermore acknowledge J. Peace and S. Debs for scientific discussion.

\section{Competing interests}

The authors declare that they have no competing interests.

\section{Availability of data and materials}

The datasets generated and analyzed during the current study are available from the corresponding author on reasonable request.

Consent for publication

Not applicable. 


\section{Ethics approval and consent to participate}

All experimental procedures were carried out as per approved protocols of the University of Sydney Animal Ethics Committee (protocol number 2015/832 and 2013/5317)

\section{Funding}

This work was funded by the National Health and Medical Research Council (\#1028241 for VC and GEG, and \#1099920 for GEG), and the Rebecca L. Cooper Research Foundation. NT was supported by the Swiss National Science Foundation (SNSF, \#P300PA_164715). The funders had no role in study design, data collection and analysis, decision to publish, or preparation of the manuscript.

\section{Publisher's Note}

Springer Nature remains neutral with regard to jurisdictional claims in published maps and institutional affiliations.

Received: 20 January 2018 Accepted: 20 April 2018 Published online: 11 May 2018

\section{References}

1. Schofield L, Grau GER. Immunological processes in malaria pathogenesis. Nat Rev Immunol. 2005;5:722-35.

2. WHO. World malaria report. Geneva: World Health Organization; 2016. p. 2016.

3. Bangirana P, Opoka RO, Bolvin MJ, Idro R, Hodges JS, John CC. Neurocognitive domains affected by cerebral malaria and severe malarial anemia in children. Learn Individ Differ. 2016:46:38-44

4. Storm J, Craig AG. Pathogenesis of cerebral malaria-inflammation and cytoadherence. Front Cell Infect Microbiol. 2014;4:100.

5. Lu F, Culleton R, Zhang M, Ramaprasad A, von Seidlein L, Zhou H, et al. Emergence of indigenous artemisinin-resistant Plasmodium falciparum in Africa. New Eng J Med. 2017;376:991-3.

6. Idro R, Marsh K, John CC, Newton CRJ. Cerebral malaria; mechanisms of brain injury and strategies for improved neuro-cognitive outcome. Pediatr Res. 2010:68:267-74.

7. Combes V, El-Assaad F, Faille D, Jambou R, Hunt NH, Grau GER. Microvesiculation and cell interactions at the brain-endothelial interface in cerebral malaria pathogenesis. Prog Neurobiol. 2010;91:140-51.

8. Hugel B, Martinez MC, Kunzelmann C, Freyssinet JM. Membrane microparticles: two sides of the coin. Physiology. 2005:20:22-7.

9. van der Heyde HC, Nolan J, Combes V, Gramaglia I, Grau GER. A unified hypothesis for the genesis of cerebral malaria: sequestration, inflammation and hemostasis leading to microcirculatory dysfunction. Trends Parasitol. 2006:22:503-8.

10. Combes V, Taylor TE, Juhan-Vague I, Mege JL, Mwenechanya J, Grau GER, et al. Circulating endothelial microparticles in Malawian children with severe falciparum malaria complicated with coma. JAMA 2004;291:2542-4.

11. Pankoui Mfonkeu JB, Gouado I, Fotso Kuaté H, Zambou O, Amvam Zollo $\mathrm{PH}, \mathrm{Grau} \mathrm{GER}$, et al. Elevated cell-specific microparticles are a biological marker for cerebral dysfunctions in human severe malaria. PLoS ONE. 2010;5:e13415.

12. Combes V, Coltel N, Albert M, van Eck M, Raymond C, Juhan-Vague I, et al. ABCA1 gene deletion protects against cerebral malaria: potential pathogenic role of microparticles in neuropathology. Am J Pathol. 2005; 166:295-302.

13. El-Assaad F, Wheway J, Hunt NH, Grau GE, Combes V. Production, fate and pathogenicity of plasma microparticles in murine cerebral malaria. PLoS Pathog. 2014;10:e1003839.

14. Penet M-F, Abou-Hamdan M, Coltel N, Cornille E, Grau GER, de Reggi M, et al. Protection against cerebral malaria by the low-molecular-weight thiol pantethine. Proc Natl Acad Sci USA. 2008;105:1321-6.

15. Yoon YJ, Kim OY, Gho YS. Extracellular vesicles as emerging intercellular communicasomes. BMB Rep. 2014:47:531-9.

16. Tiberti N, Latham SL, Bush S, Cohen A, Opoka RO, John CC, Juillard A, Grau GE, Combes V. Exploring experimental cerebral malaria pathogenesis through the characterisation of host-derived plasma microparticle protein content. Sci Rep. 2016:6:37871.
17. Fleissner F, Goerzig Y, Haverich A, Thum T. Microvesicles as novel biomarkers and therapeutic targets in transplantation medicine. Am J Transplant. 2012:12:289-97.

18. Bartel DP. MicroRNAs: genomics, biogenesis, mechanism, and function. Cell. 2004;116:281-97.

19. Liang H, Zen $K$, Zhang J, Zhang C-Y, Chen X. New roles for microRNAs in cross-species communication. RNA Biol. 2013;10:367-70.

20. Hunter MP, Ismail N, Zhang X, Aguda BD, Lee EJ, Lianbo Y, et al. Detection of microRNA expression in human peripheral blood microvesicles. PLOS ONE. 2008;3:e3694.

21. Collino F, Deregibus MC, Bruno S, Sterpone L, Aghemo G, Viltono L, et al. Microvesicles derived from adult human bone marrow and tissue specific mesenchymal stem cells shuttle selected pattern of miRNAs. PLOS ONE. 2010:5:e11803.

22. Hakimi MA, Cannella D. Apicomplexan parasites and subversion of the host cell microRNA pathway. Trends Parasitol. 2011;27:481-6.

23. Delic D, Dkhil M, Al-Quraishy S, Wunderlich F. Hepatic miRNA expression reprogrammed by Plasmodium chabaudi malaria. Parasitol Res. 2011;108:1111-21.

24. El-Assaad F, Hempel C, Combes V, Mitchell AJ, Ball HJ, Kurtzhals JAL, et al. Differential microRNA expression in experimental cerebral and noncerebral malaria. Infect Immun. 2011;79:2379-84.

25. Chamnanchanunt S, Kuroki C, Desakorn V, Enomoto M, Thanachartwet $V$, Sahassananda D, et al. Downregulation of plasma miR-451 and miR16 in Plasmodium vivax infection. Ex Parasitol. 2015:155:19-25.

26. Barker KR, Lu Z, Kim H, Zheng Y, Chen J, Conroy AL, et al. miR-155 modifies inflammation, endothelial activation and blood-brain barrier dysfunction in cerebral malaria. Mol Med. 2017;23:24-33.

27. Mantel PY, Hjelmqvist D, Walch M, Kharoubi-Hess S, Nilsson S, Ravel D, et al. Infected erythrocyte-derived extracellular vesicles alter vascular function via regulatory Ago2-miRNA complexes in malaria. Nat Commun. 2016:7:12727.

28. Moro L, Bardaji A, Macete E, Barrios D, Morales-Prieto DM, Espana C, et al. Placental microparticles and microRNAs in pregnant women with Plasmodium falciparum or HIV infection. PLOS ONE. 2016;11:e0146361.

29. Yang Y, Liu Q, Lu J, Adah D, Yu S, Zhao S, et al. Exosomes from Plasmodium-infected hosts inhibit tumor angiogenesis in a murine Lewis lung cancer model. Oncogenesis. 2017:6:e351.

30. Al-Quraishy S, Dkhil MA, Delic D, Abdel-Baki AA, Wunderlich F. Organ-specific testosterone-insensitive reponse of miRNA expression of C57BL/6 mice to Plasmodium chabaudi malaria. Parasitol Res. 2012:111:1093-101.

31. Cohen A, Combes V, Grau GE. MicroRNAs and malaria—a dynamic interaction still incompletely understood. J Neuroinfect Dis. 2015;6:165.

32. Dkhil MA, Al-Quraishy SA, Abdel-Baki AS, Delic D, Wunderlich F. Differential miRNA expression in the liver of Balb/c mice protected by vaccination during crisis of Plasmodium chabaudi blood-stage malaria. Front Microbiol. 2017;7:2155

33. Mead EA, Tu Z. Cloning, characterization, and expression of microRNAs from the Asian malaria mosquito, Anopheles stephensi. BMC Genomics. 2008;9:244

34. Xue X, Zhang Q, Huang Y, Feng L, Pan W. No miRNA were found in Plasmodium and the ones identified in RBCs could not be correlated with infection. Malar J. 2008;7:47.

35. La Monte G, Philip N, Reardon J, Lacsina JR, Majoros W, Chapman L, et al. Translocation of sickle cell erythrocyte microRNAs into Plasmodium falciparum inhibits parasite translation and contributes to malaria resistance. Cell Host Microbe. 2012;12:187-99.

36. Jain S, Rana V, Shrinet J, Sharma A, Tridibes A, Sunil S. Blood feeding and Plasmodium infection alters the miRNome of Anopheles stephensi. PLoS ONE. 2014;9:e98402.

37. Rae C, McQuillan JA, Parekh SB, Bubb WA, Weiser S, Balcar VJ, et al. Brain gene expression, metabolism, and bioenergetics: interrelationships in murine models of cerebral and noncerebral malaria. FASEB J. 2004;18:499-510.

38. Grau GE, Piguet PF, Engers HD, Louis JA, Vassalli $P$, Lambert PH. I3t4+ T lymphocytes play a major role in the pathogenesis of murine cerebral malaria. J Immunol. 1986;137:2348-54.

39. Potter SM, Chan-Ling T, Rosinova E, Ball HJ, Mitchell AJ, Hunt NH. A role for Fas-Fas ligand interactions during the late-stage neuropathological 
processes of experimental cerebral malaria. J Neuroimmunol. 2006;173:96-107.

40. Mestdagh P, Van Vlierberghe P, De Weer A, Muth D, Westermann F, Speleman F, et al. A novel and universal method for microRNA RT-qPCR data normalization. Genome Biol. 2009;10:R64.

41. Vlachos IS, Zagganas K, Paraskevopoulou MD, Georgakilas G, Karagkouni D, Vergoulis T, et al. DIANA-miRPath v3.0: deciphering microRNA function with experimental support. Nucleic Acids Res. 2015;43:W460-6.

42. Sethupathy P, Megraw M, Hatzigeorgiou AG. A guide through present computational approaches for the identification of mammalian microRNA targets. Nat Methods. 2006;3:881-6.

43. Kanehisa M, Goto S. KEGG: kyoto encyclopedia of genes and genomes. Nucleic Acids Res. 2000;28:27-30.

44. Benz F, Roderburg C, Vargas Cardenas D, Vucur M, Gautheron J, Koch A, et al. $U 6$ is unsuitable for normalization of serum miRNA levels in patients with sepsis or liver fibrosis. Exp Mol Med. 2013;45:e42.

45. Roberts TC, Coenen-Stass AML, Wood MJA. Assessment of RT-qPCR normalization strategies for accurate quantification of extracellular microRNAs in murine serum. PLoS ONE. 2014;9:e89237.

46. Eriksen AH, Andersen RF, Pallisgaard N, Sorensen FB, Jakobsen A, Hansen TF. MicroRNA expression profiling to identify and validate reference genes for the relative quantification of microRNA in rectal cancer. PLoS ONE. 2016;11:e0150593.

47. Lackner P, Burger C, Pfaller K, Heussler V, Helbok R, Morandell M, et al. Apoptosis in experimental cerebral malaria: spatial profile of cleaved caspase-3 and ultrastructural alteration in different disease stages. Neuropathol Appl Neurobiol. 2007;33:560-71.

48. Sheedy FJ, O'Neill LA. Adding fuel to fire: microRNAs as a new class of mediators of inflammation. Ann Rheum Dis. 2008;67(Suppl 3):iii50-5.

49. Sonkoly E, Stahle M, Pivarcsi A. MicroRNAs and immunity. novel players in the regulation of normal immune function and inflammation. Semin Cancer Biol. 2008;18:131-40.

50. Grau GER, Fjardo LF, Piguet PF, Allet B, Lambert PH, Vassalli P. Tumor necrosis factor (cachectin) as an essential mediator in murine cerebral malaria. Science. 1987:237:1210-2.

51. Quinn SR, O'Neill LA. A trio of microRNAs that control Toll-like receptor signalling. Int Immunol. 2011;23:421-5.
52. Giza DE, Vasilescu C. MicroRNA's role in sepsis and endotoxin tolerance. More players on the stage. Chirurgia. 2010;105:625-30.

53. Bellingham SA, Coleman BM, Hill AF. Small RNA deep sequencing reveals a distinct miRNA signature released in exosomes from prion-infected neuronal cells. Nucleic Acids Res. 2012:40:10937-49.

54. O'Connell RM, Rao DS, Baltimore D. microRNA regulation of inflammatory responses. Annu Rev Immunol. 2012;30:295-312.

55. Zhong Q, Wang T, Lu P, Zhang R, Zou J, Yuan S. miR-193b promotes cell proliferation by targeting Smad3 in human glioma. J Neurosci Res. 2014;95:619-26.

56. Eigsti RL, Sudan B, Wilson ME, Graff JW. Regulation of activation-associated microRNA accumulation rates during monocyte-to-macrophage differentiation. J Biol Chem. 2014;289:28433-47.

57. Braconi C, Valeri N, Gasparini P, Huang N, Taccioli C, Nuovo G, et al. Hepatitis $C$ virus proteins modulate microRNA expression and chemosensitivity in malignant hepatocytes. Clin Cancer Res. 2010;16:957-66.

58. Li C, Chen Y, Chen X, Wei Q, Cao B, Shang H. Downregulation of microRNA-193b-3p promotes autophagy and cell survival by targeting TSC1/ mTOR signaling in NSC-34 cells. Front Mol Neurosci. 2017;10:160.

59. Wang HJ, Zhang PJ, Chen WJ, Feng D, Jia YH, Xie LX. Four serum microRNAs identified as diagnisotic biomarkers of sepsis. J Trauma Acute Care Surg. 2012;73:850-4.

60. Liang Y, Ridzon D, Wong L, Chen C. Characterization of microRNA expression profiles in normal human tissues. BMC Genomics. 2007:8:166.

61. Weber JA, Baxter DH, Zhang S, Huang DY, Huang KH, Lee MJ, et al. The microRNA spectrum in 12 body fluids. Clin Chem. 2010;56:1733-41.

62. Zhao K, Liang G, Sun X, Guan LL. Comparative miRNAome analysis revealed different miRNA expression profiles in bovine sera and exosomes. BMC Genomics. 2016;17:630.

63. Alexander M, Hu R, Runtsch MC, Kagele DA, Mosbruger TL, Tolmachova T, et al. Exosome-delivered microRNAs modulate the inflammatory resposne to endotoxin. Nat Commun. 2015;6:7321.

64. Miller LH, Baruch DI, Marsh K, Doumbo OK. The pathogenic basis of malaria. Nature. 2002;415:673-9.

65. Marques-Rocha JL, Samblas M, Milagro FI, Bressan J, Martinez JA, Marti A. Noncoding RNAs, cytokines, and inflammation-related diseases. FASEB J. 2015;29:3595-611.
Ready to submit your research? Choose BMC and benefit from:

- fast, convenient online submission

- thorough peer review by experienced researchers in your field

- rapid publication on acceptance

- support for research data, including large and complex data types

- gold Open Access which fosters wider collaboration and increased citations

- maximum visibility for your research: over $100 \mathrm{M}$ website views per year

At BMC, research is always in progress.

Learn more biomedcentral.com/submissions 